\title{
A New Resolution Inference by Petri Net
}

\author{
Shifen Xia ${ }^{1}$ Yingbin Qin ${ }^{1}$ Yang $\mathrm{Xu}^{2}$ \\ ${ }^{1}$ Department of Mathematics, Southwest Jiaotong University, Chengdu 610031, P.R. China \\ ${ }^{2}$ Intelligent Control Development Center, Southwest Jiaotong University, Chengdu 610031, P.R. China
}

\begin{abstract}
This paper gives a method of resolution inference by Petri net, which is different from the T-invariant. Only through reducing the structure of Petri net model step by step, the resolution inference can be accomplished easily. We have discussed the inference from Horn clause set to first-order one. All the computations of inference are finished in polynomial time.
\end{abstract}

Keywords: Resolution inference, Petri net, T-invariant

\section{Introduction}

Methods of analysis for Petri nets can be classified into following three groups: 1) the cover ability (reachability) tree method, 2) the matrix-equation approach, and 3) reduction or decomposition techniques. The first method involves essentially the enumeration of all reachable markings or their coverable markings. It should be able to apply to all classes of nets, but is limited small nets duo to the complexity of the state - space exposition. On the other hand, matrix equation and reduction techniques are powerful but in many cases they are applicable only to special subclasses of Petri nets or special situations. Although many techniques have been proposed for analysis of Petri nets, there is an inherent difficulty of complexity in using them for real-life application. To copy with this problem, we propose a method of resolution inference through cutting the Petri net model of clauses, which can reduce the complexity of the state- space. We can get better results than in the method of T-invariant, and the process of inference can also be showed directly. The method is not only fit for Horn clause set, usual clause set, but also first-order one, the computing complexity is polynomial time.

The rest of the paper is organized as fallows: In section 2 the knowledge about the paper is described. Section 3 describes a cutting resolution principal based on the Petri net for the propositional logic, the completeness of the resolution is proved. The extend cutting algorithm for first-order logic contains in section 4 . The conclusion is given in section 5 .

\section{Related work}

Definition 1[1] A Place/Transition Petri net model of propositional logic is a 5-tuple (P,T,F, $\left.\mathrm{M}_{0}, \mathrm{~W}\right)$ where: $\mathrm{P}$ is a finite set of places, $\mathrm{T}$ is a finite set of transitions, $\mathrm{F}$ is a set of arcs, $\mathrm{F} \subseteq(\mathrm{P} \times \mathrm{T}) \cup(\mathrm{T} \times \mathrm{P}) ; \mathrm{W}: \mathrm{F} \rightarrow\{1\}$ is a weight function, $\mathrm{M}_{0}: \mathrm{P} \rightarrow \mathrm{N}$ is the initial marking, $\mathrm{P} \cap \mathrm{T}=\phi$ (null set), $\quad \mathrm{P} \cup \mathrm{T} \neq \phi$.

Definition 2[2] A Predicate/Transition Petri net model of first-order predicate logic is a 9-tuple ( $\mathrm{P}$, T,F,D,V, $A_{P}, A_{T}, A_{F}, M_{0}$ ) where: (1)(P,T,F)is the basic net defined in above; (2)D is a nonempty and finite set, which is called the individual set of the $\sum, \Omega$ is the set of operators for $\mathrm{D}$; (3) V is the set of individual variables over $D$;(4) $A_{p}: P \rightarrow \Pi$, where $\Pi$ is the set of changeable predicate $\forall \mathrm{p} \in \mathrm{P}$, if $\mathrm{A}_{\mathrm{P}}(\mathrm{p})$ is a n-ary predicate, then $\mathrm{p}$ is called n-ary predicate ; $(5) \mathrm{A}_{\mathrm{T}}: \mathrm{T} \rightarrow$ $f_{D}$, where $f_{D}$ is the formula set on D. $\forall \mathrm{t} \in \mathrm{T}, \mathrm{A}_{\mathrm{T}}(\mathrm{t})$ can only be a static predicate or an operator in $\Omega$; (6) $\mathrm{A}_{\mathrm{F}}: \mathrm{F} \rightarrow f_{S}$, where $f_{S}$ is the symbolic sum on $\mathrm{D}$, $\forall \mathrm{p} \in \mathrm{P}$, if $(\mathrm{t}, \mathrm{p}) \in \mathrm{F}$ or $(\mathrm{p}, \mathrm{t}) \in \mathrm{F}, \mathrm{A}_{\mathrm{F}}(\mathrm{t}, \mathrm{p})$ or $\mathrm{A}_{\mathrm{F}}(\mathrm{p}, \mathrm{t})$ is a n-ary symbolic sum, otherwise, $A_{F}(t, p)$ or $A_{F}(p, t) e q u a l$ to null; (7) $\mathrm{M}_{0}: \mathrm{P} \rightarrow f_{S}, \mathrm{M}_{0}(\mathrm{p})$ is the sum of n-ary predicate.

Theorem 1[3] If the ground clause set $S$ is unsatisfiability. then Petri net of $\mathrm{S}$ has T-invariant $\mathrm{X}$, such that $X \geq 0$.

Theorem 2[3] Horn ground clause set $\mathrm{S} \cup \neg \mathrm{G}$ is unsatisfiability iff Petri net of $\mathrm{S} \cup \neg \mathrm{G}$ has T-invariant $X$, such that $X\left(t_{g}\right) \geq 0$, where $t_{g}$ is the goal transition.

Theorem 3[3] The first-order clauses set $S$ $\cup \neg \mathrm{G}$ is unsatisfiability iff Petri net of $\mathrm{S} \cup \neg \mathrm{G}$ has T-invariant $\mathrm{X}$, such that $\mathrm{X}\left(\mathrm{t}_{\mathrm{g}}\right) \neq \phi$, where $\mathrm{t}_{\mathrm{g}}$ is the goal transition.

\section{Cutting resolution principal}

\subsection{Cutting resolution principal of Horn ground clause set}

Let HPNM be the Petri net model of Horn ground clause set $\mathrm{S}[4], *_{\mathrm{t}}=\{\mathrm{p} \mid \mathrm{p} \in \mathrm{P},(\mathrm{p}, \mathrm{t}) \in \mathrm{F}\}, \mathrm{t}^{*}=\{\mathrm{p} \mid \mathrm{p} \in \mathrm{P},(\mathrm{t}$, $\mathrm{p}) \in \mathrm{F}\}$. Here, we define null transition to express the null clause and isolated place. 
Definition 3 The transition $t$ is called null transition noted - if ${ }^{*} \mathrm{t}=\mathrm{t}^{*}=\phi$ (null set); $\mathrm{p}$ be isolated place if $\left.\right|^{*} \mathrm{p}|=| \mathrm{p}^{*} \mid=0$.

Let ${ }^{*} \mathrm{p}=\{\mathrm{t} \mid \mathrm{t} \in \mathrm{T}, \quad(\mathrm{t}, \mathrm{p}) \in \mathrm{F}\}, \quad \mathrm{p}^{*}=\{\mathrm{t} \mid \mathrm{t} \in \mathrm{T}$, $(p, t) \in F$, and $|G|$ be the element number in set $G$, and suppose the source transitions are always reachable.

Algorithm I Let $\mathrm{p}$ be a place, the resolution principal of Horn clause set include the following three process:

(1) If $\left.\right|^{*} p|=k(k \geq 1),| p^{*} \mid=m(m \geq 1)$, and there is at least a mark in places $\mathrm{p}$, let $* \mathrm{p}=\left\{\mathrm{t}_{1}, \mathrm{t}_{2}, \cdots, \mathrm{t}_{\mathrm{k}}\right\}, \mathrm{p}^{*}=$ $\left\{\mathrm{t}_{1}, \mathrm{t}_{2}, \cdots, \mathrm{t}_{\mathrm{m}}\right\}$,then cut the place $\mathrm{p}$ and all arcs $\left(t_{i}, p\right)(i=1,2, \cdots, k)$ and $\left(p, t_{j}\right)(j=1,2, \cdots, m)$, combine the transitions $t_{i}$ and $t_{j}$ be transition $t_{i j}(i=1,2, \cdots, k$; $\mathrm{j}=1,2, \cdots, \mathrm{m})$, put a mark in the place $\left(\mathrm{t}_{\mathrm{ij}}\right)^{*}$;

(2) If $\mathrm{t}^{*} \subseteq{ }^{*} \mathrm{t}$ or ${ }^{*} \mathrm{p}=\mathrm{p}^{*}$ (i.e. $\mathrm{t}$ express tautology), then cut the transition $\mathrm{t}$ and its all relation of arcs;

(3) if $|* p|=\left|p^{*}\right|=0$, then cut the place $p$.

Some notes:

i) In the process (1), $t_{i j}$ is corresponding to the resolent of clause $t_{i}$ and $t_{j}$, process (2) to reducing the tautology, process (3) to be cutting isolated place which is produced by above process and is no efficient to resolution.

ii) The lower sign ij in $t_{i j}$ is to remember the process of resolution inference. If we note the lower sign $i j$ to be set $\{i, j\}$, then there is a process of replace the subset $\{\mathrm{i}\}$ or $\{\mathrm{j}\}$ to be $\{\mathrm{i}, \mathrm{j}\}$.

Let CHPNM be the model after applied the any process of above for the model HPNM.

Example 1 Let $\mathrm{S}=\{\mathrm{A}, \neg \mathrm{A} \vee \mathrm{B}\}$, then the HPNM and CHPNM are in the follow:

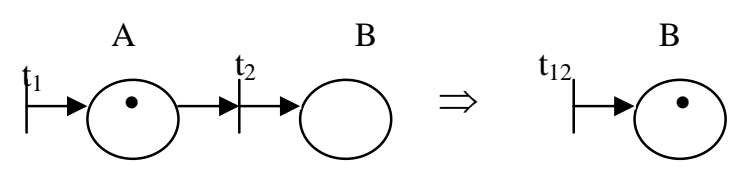

Fig. 1: Models of example 1.

Theorem 4 If $\mathrm{S}$ is a Horn clause set, then $\mathrm{S}$ is unsatisfiability iff there is the null transition - in the model of CHPNM .

Proof: If there is null transition - in CHPNM, because the transition - express null clause, so the conclusion is hold. On the other hand, if Horn clause set $S$ is unsatisfiability, because the all process in cutting resolution principal is the process of resolution, and unit resolution is completeness for Horn clause, so in every model of CHPNM, we can chose the place of unit clause to be cutting literal, after finite process, we can get null clause, i.e. null transition.

Example 2 [5]Let $\mathrm{S}=\{\mathrm{A}, \mathrm{B}, \mathrm{A} \wedge \mathrm{B} \rightarrow \mathrm{C}, \mathrm{B} \wedge \mathrm{C} \rightarrow$ $\mathrm{D}, \mathrm{D} \rightarrow \mathrm{A}, \mathrm{D} \rightarrow \mathrm{C}\}, \mathrm{G}=\mathrm{C} \wedge \mathrm{D}$, then the HPNM and
SHPNM of $\mathrm{S} \cup \neg \mathrm{G}$ are in follow:
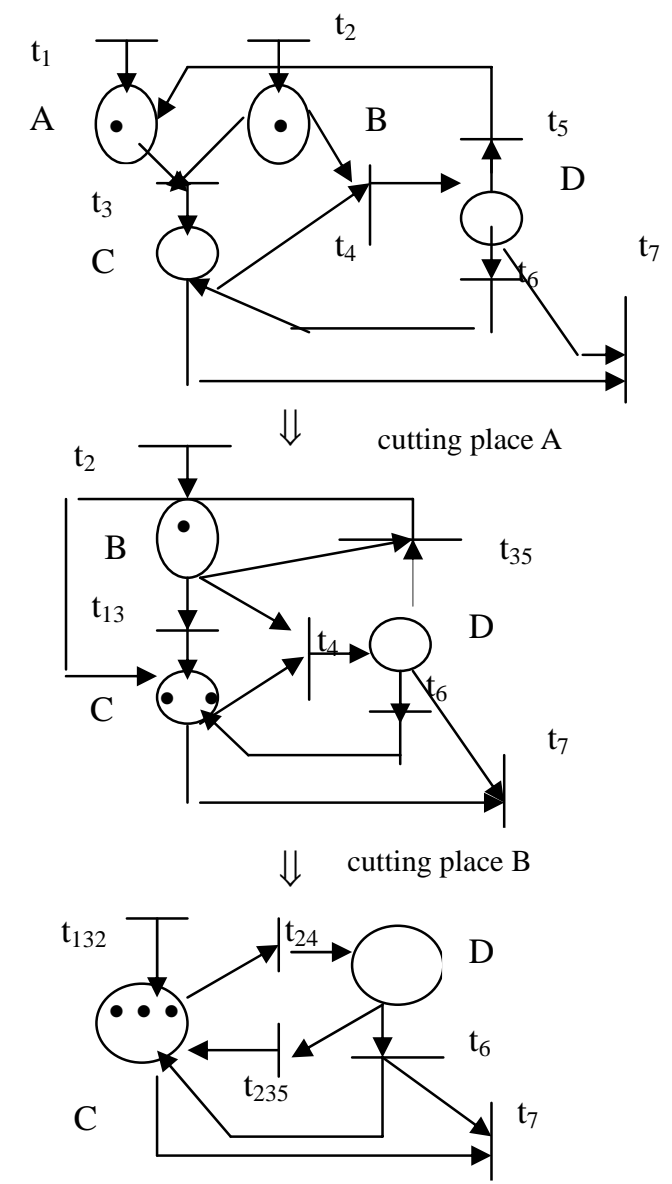

$\Downarrow \quad$ cutting place $\mathrm{C}$

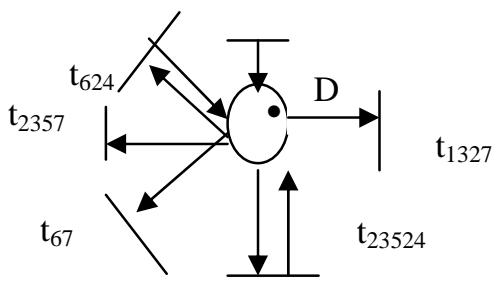

$\Downarrow$ cutting tautology

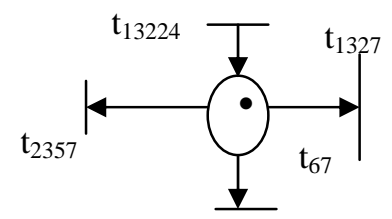

$\Downarrow \quad$ cutting place D

$\mathrm{t}_{13224} \quad \mathrm{t}_{132241327} \quad \mathrm{t}_{132242357}$

Fig.2: Models of example 2.

In the end, we get three null transitions 
$\mathrm{t}_{1322467}, \mathrm{t}_{132241327}$ and $\mathrm{t}_{132242357}$, So $\mathrm{s} \cup \neg \mathrm{G}$ is unsatisfiability that is to say $\mathrm{G}$ can be get from clause set $\mathrm{S}$.

Let $\mathrm{X}_{\mathrm{i}}$ be the vector consisted of frequency in the lower sign array of null transition, then $X_{1}=(1,2,1,1,0,1,1), \quad X_{2}=(2,3,2,1,0,0,1), \quad X_{3}=$ $(1,3,2,1,1,0,1)$ in example 2 , which is corresponding to T-invariants[5] $: \mathrm{T}_{1}=\left(2,4,2,2,0,1,1, \mathrm{~T}_{2}=(2,3,2,1,0,0,1), \mathrm{T}_{3}\right.$ $=(2,5,3,2,1,0,1))$, the other two T-invariants: $\mathrm{T}_{4}$ $=(0,1,0,1,0,1,0), T_{5}=(0,2,1,1,1,0,0)$ have nothing to do with satisfiability, so there is no $\mathrm{X}_{\mathrm{i}}$ corresponding them. In the sense of this, we say that our method is simple than the method of T-invariants.

Let $\mathrm{X}$ be the set of vectors consisted of frequency in the lower sign array of null transition in model of SHPNM, $\mathrm{T}$ be the set of all T-invariants in model, we can also get improve results as followings (all the proves are canceled):

Theorem 5 If $X_{i} \in X$, then there is $T_{j} \in T$,such that $0 \leq X_{i} \leq T_{j}$.

Theorem 6 If $X_{i} \in X, T_{j} \in T$, and $X_{i} \leq T_{j}, T_{j}\left(t_{g}\right)$ $\geq 0$, then $X_{i}\left(t_{g}\right) \geq 0$, where $t_{g}$ is the goal transition.

Theorem $7 \mathrm{~S}$ is satisfiable and $\mathrm{S} \cup \neg \mathrm{G}$ is unsatisfiable iff there is $X_{i} \in X$, such that $X_{i}\left(t_{g}\right) \geq 0$, where $t_{g}$ is the goal transition.

Theorem 8 Let $\mathrm{S}$ be Horn clause set, $\mathrm{S}$ is unsatisfiable iff $\mathrm{X} \neq \phi$.

\subsection{Cutting resolution principal of usual ground clause set}

We have given the cutting resolution principal for Horn clause set only based on the structure of Petri net; here we extend the principal to usual clause set and directly express the Petri net model of usual clause.

3.2.1. Petri net model of usual ground clause set

We can classify the composite rules into four rule types as follows:

Type 1: $\mathrm{A}_{1} \wedge \mathrm{A}_{2} \wedge \cdots \wedge \mathrm{A}_{\mathrm{p}} \rightarrow \mathrm{B}_{1} \vee \mathrm{B}_{2} \vee \cdots \vee \mathrm{B}_{\mathrm{q}}$;

Type $2: A_{1} \wedge A_{2} \wedge \cdots \wedge A_{p} \rightarrow B_{1} \wedge B_{2} \wedge \cdots \wedge B_{q}$;

Type $3: A_{1} \vee A_{2} \vee \cdots \vee A_{p} \rightarrow B_{1} \vee B_{2} \vee \cdots \vee B_{q}$;

Type $4: A_{1} \vee A_{2} \vee \cdots \vee A_{p} \rightarrow B_{1} \wedge B_{2} \wedge \cdots \wedge B_{q}$.

The representations of all type by Petri net shows in follow:
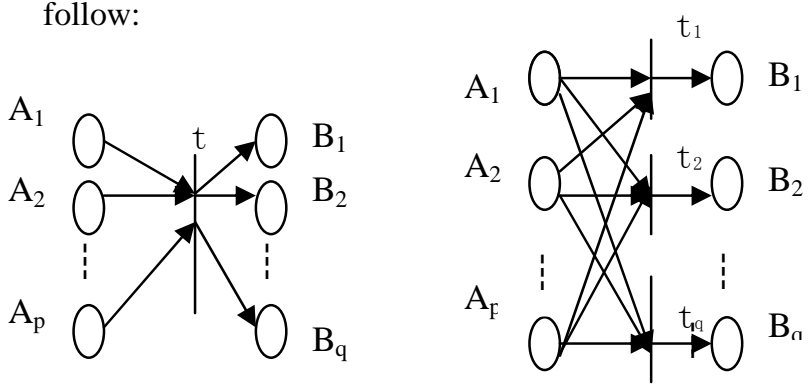

Petri net model of type 1 Petri net model of type 2
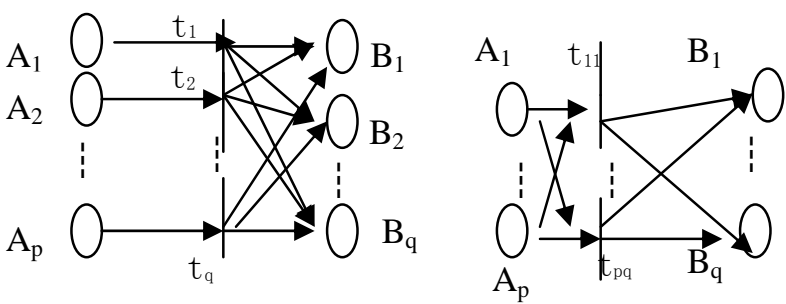

Petri net model of type 3 Petri net model of type 4 Fig. 3: Models of four rules.

There are no negative literals in the above rules. In fact, how to represent the rule which has negative literal is key to model the usual clause set[6], here we represent the rule as $A_{1} \wedge A_{2} \rightarrow \neg B_{1} \vee B_{2}$ to be following way because $A_{1} \wedge A_{2} \rightarrow \neg B_{1} \vee B_{2}=\neg A_{1} \vee$ $\neg \mathrm{A}_{2} \vee \neg \mathrm{B}_{1} \vee \mathrm{B}_{2}=\mathrm{A}_{1} \wedge \mathrm{A}_{2} \wedge \mathrm{B}_{1} \rightarrow \mathrm{B}_{2}$.

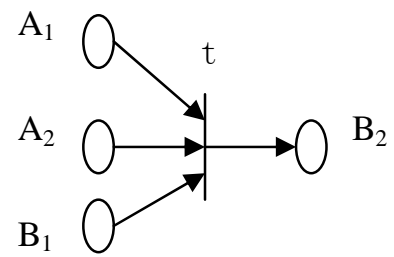

Fig. 4: Model of $A_{1} \wedge A_{2} \rightarrow \neg B_{1} \vee B_{2}$.

\subsection{Cutting resolution principal}

Algorithm II Let $\mathrm{p}$ be a place and has at least a mark, the cutting resolution principal include the following three process:

(1) If $\left.\right|^{*} \mathrm{p}|=\mathrm{k}(\mathrm{k} \geq 1),| \mathrm{p}^{*} \mid=\mathrm{m}(\mathrm{m} \geq 1)$, and there is at least a mark in places $\mathrm{p}$, let ${ }^{*} \mathrm{p}=\left\{\mathrm{t}_{1}, \mathrm{t}_{2}, \cdots, \mathrm{t}_{\mathrm{k}}\right\}, \mathrm{p}^{*}=$ $\left\{\mathrm{t}_{1}, \mathrm{t}_{2}, \cdots, \mathrm{t}_{\mathrm{m}}\right\}$,then cut the place $\mathrm{p}$ and all arcs $\left(t_{i}, p\right)(i=1,2, \cdots, k)$ and $\left(p, t_{j}\right)(j=1,2, \cdots, m)$,combine the transitions $t_{i}$ and $t_{j}$ be transition $t_{i j}(i=1,2, \cdots, k$; $\mathrm{j}=1,2, \cdots, \mathrm{m})$, put a mark in the place $\left(\mathrm{t}_{\mathrm{ij}}\right)^{*}$;

(2) If $\mathrm{t}^{*} \cap * \mathrm{t} \neq \Phi$ (i.e. $\mathrm{t}$ represent tautology), then cut the transition $t$ and its all relation of arc;

(3) if $\left.\right|^{*} p \mid=0$ and $\left|p^{*}\right| \neq 0$ or $\left|{ }^{*} p\right| \neq 0$ and $\left|p^{*}\right|=0$, then cut all the transitions and arcs in *p (rule of pure literal), otherwise cut the place $\mathrm{p}$.

Let UPNM be the Petri net model of usual clause set, CUPNM be the model after applied the any process of above for UPNM

Theorem 9 If there are finite literals in usual ground clause set $\mathrm{S}$, then $\mathrm{S}$ is unsatisfiability iff there is the null transition - in the model of CUPNM .

Let $\mathrm{X}$ be the set of vectors consisted of frequency in the lower sign array of null transition in model of CUPNM\}

Theorem 10 Let $\mathrm{S}$ be usual ground clause set, then $S$ is unsatisfiable iff $X \neq \phi$.

Example 3 Let $\mathrm{S}=\{\mathrm{A}, \mathrm{B}, \mathrm{A} \wedge \mathrm{B} \rightarrow \neg \mathrm{C} \vee \mathrm{D}, \mathrm{D} \rightarrow \mathrm{E}$ 
$\wedge \mathrm{F}, \neg \mathrm{C} \rightarrow \mathrm{F} \vee \mathrm{H}\}, \mathrm{G}=\mathrm{E} \wedge \mathrm{H}$, then the model of UPNM and CUPNM is as follows:

A

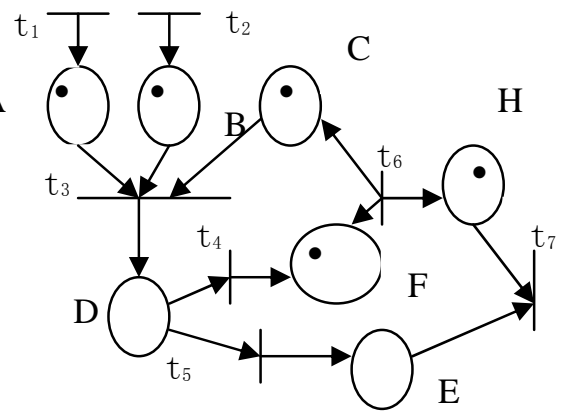

$\Downarrow \quad$ cut place $\mathrm{A}$

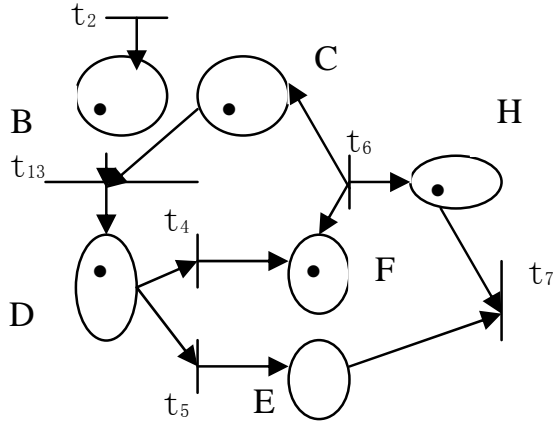

$\Downarrow \quad$ cut place B

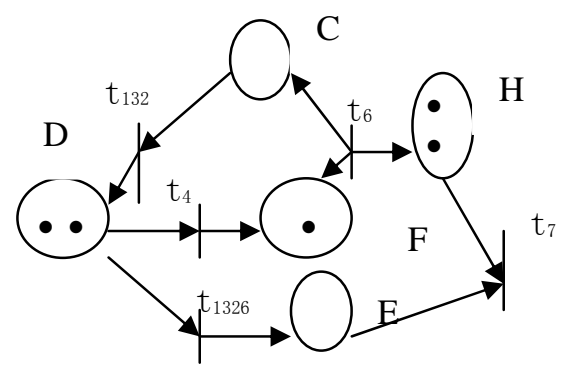

$\Downarrow$

cut place $\mathrm{C}$

$\mathrm{D}$

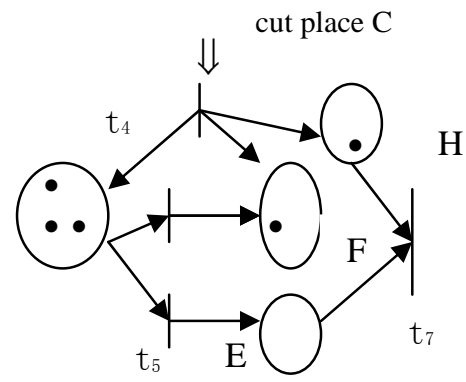

$\Downarrow$

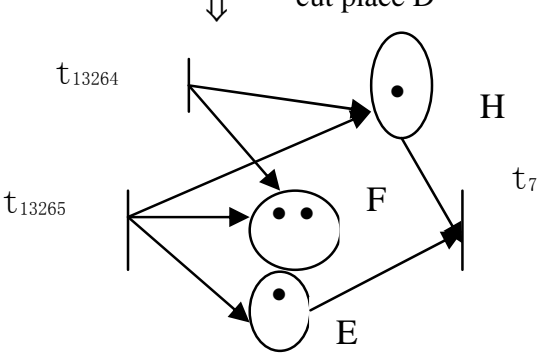

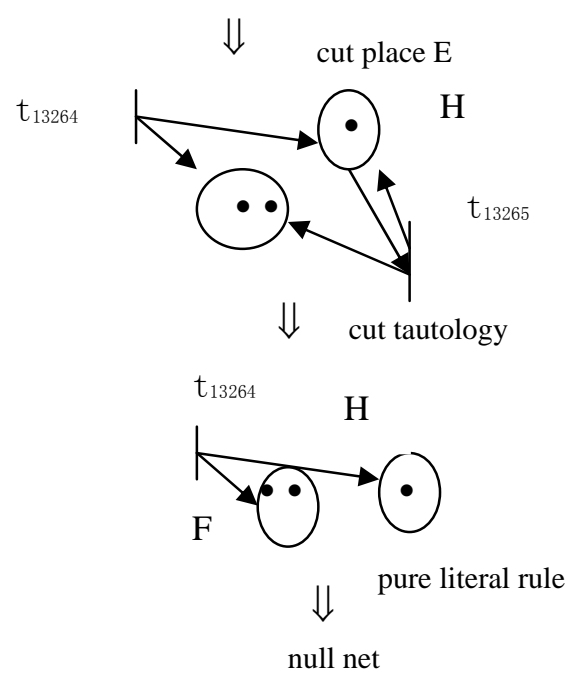

Fig.5: Models of example 3.

So $S \cup \neg G$ is unsatisfiability for $X=\phi$. For this example, there is a question that we change the clause $\mathrm{t}_{6}(\neg \mathrm{C} \rightarrow \mathrm{F} \vee \mathrm{H})$ to be a source transition while it isn't a source one, this will reinforce the condition for clause set $\mathrm{S}$, how to explain this? We think this will not effect the resolution of usual clause set for we only use the structure of Petri net model not using the method of T-invariant.

\section{Cutting resolution principal of first-order logic}

A first-order clause is called Horn clause if there is at most one positive literal in the clause, otherwise called usual clause. If a set is only consisted of Horn clauses, then call it first-order Horn clause set. We can model first-order clause set in Petri net according to define 2, the cutting resolution principal is similar to the above, the only difference is that we must apply most general unifier (mgu) to two transitions and replace the corresponding weights connected the place $\mathrm{p}$ we cut. We don't give the cutting resolution principal of first-order clause set, only explain it with example to shrink the length of the paper.

Example 4 Let $\mathrm{S}=\{\mathrm{P}(\mathrm{a}), \mathrm{Q}(\mathrm{b}), \mathrm{D}(\mathrm{b}), \neg \mathrm{D}(\mathrm{y}) \bigvee$ $\mathrm{L}(\mathrm{a}, \mathrm{y}), \neg \mathrm{P}(\mathrm{x}) \bigvee \neg \mathrm{Q}(\mathrm{y}) \vee \neg \mathrm{L}(\mathrm{x}, \mathrm{y})$, the Petri net model of $\mathrm{S}$ and its cutting inference are as follows:

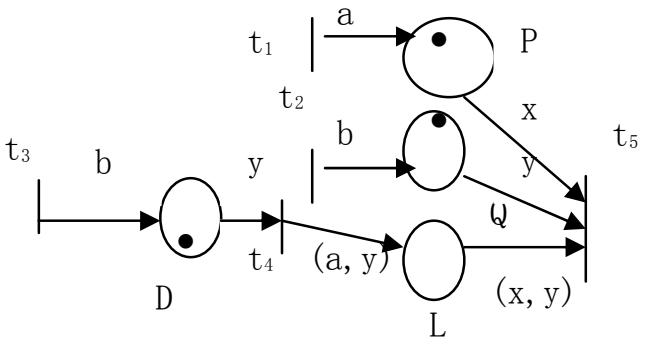



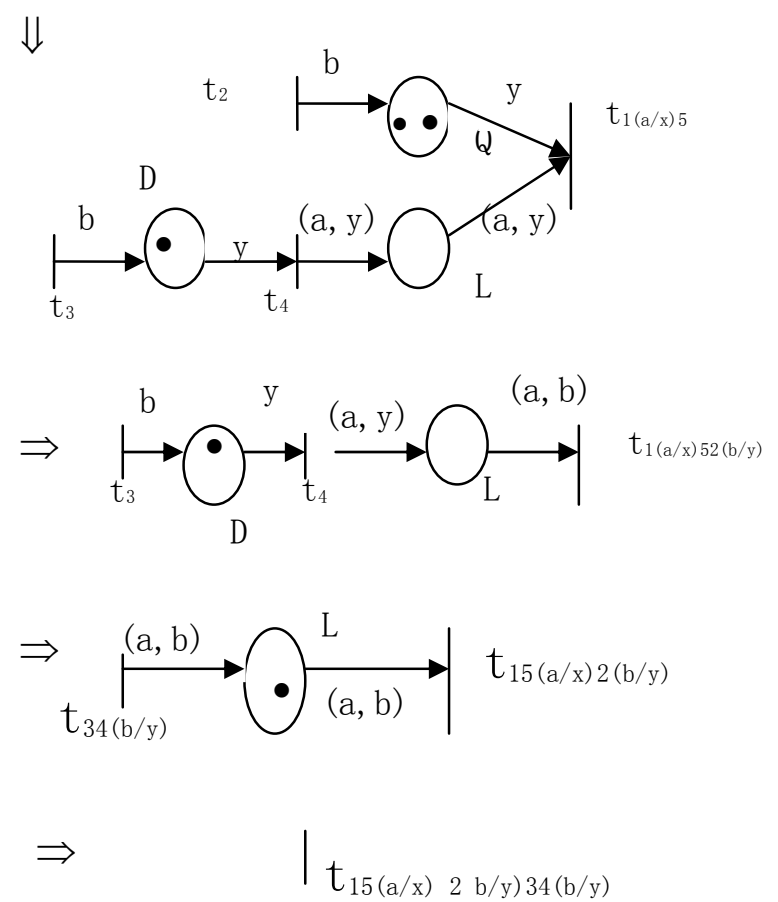

Fig. 6: Models of example 4.

$S$ is is unsatisfiability for getting null transition.

\section{Conclusions}

This paper studies the cutting resolution principal based on the Petri net model of propositional logic and first-order predicate logic. We chose the resolution literal (i.e. cutting literal) through the mark of place. The lower sign of transition can show the whole process of the inference, and the computing complexity is polynomial time if there have only finite literals in clause set $\mathrm{S}$.

\section{Acknowledgement}

This work is partially supported by National Nature Science Foundation of China (Grant No. 60474022) and School Foundation of South West Jiaotong University (Grant No. 2006B09).

\section{References}

[1] T. Murata, Petri nets: Properties, Analysis and Applications Proceedings of IEEE, 77(4): 541-579, 1989.

[2] H. Genrich, System modeling with high-level
Petri nets, Theoret. Comput. Sci. 13: 109-136, 1981.

[3] A. Sinachopoulos, Derivation of a contradiction by resolution using Petri nets, Petri Net Newsletter, 26: 16-29, 1998.

[4] M. Murata, A Petri net model for reasoning in the presence of inconsistency, IEEE Trans. On Knowledge and Data Engineering , 3( 3): 281-191, 1991.

[5] C. Lin, Logical Inference of Horn Clauses in Petri Net Models, IEEE Trans. Knowledge and Data Engineering, 5(3): 416-424, 1993.

[6] C. Lin, Logical inference Clauses in Petri Net Models Using T-invariants, Chinese $J$. Computers , 19(10): 762-767,1996. 